\title{
Caractérisation et traitabilité des sous-produits solides de l'assainissement pluvial
}

\author{
par Cécile Delattre, Jean-Luc Bertrand-Krajewski \\ GARIH/CTIA Lyonnaise des Eaux
}

\section{I $\square$ INTRODUCTION}

Le terme "sous-produits solides de l'assainissement " regroupe l'ensemble des solides liés à l'entretien et à l'exploitation des réseaux d'assainissement et des voiries : produits de balayage, de curage des avaloirs et des collecteurs, produits de décantation en bassin, solides des chambres à sable, etc., dans le cadre d'une approche intégrée des rejets urbains de temps de pluie. Actuellement, les sousproduits solides de l'assainissement sont, pour la plupart. mis en décharge où leur admission rencontrera des difficultés croissantes. Le respect de la réglementation, et plus parti- culièrement de la loi sur les déchets du 13 juillet 1992 qui n'autorisera la mise en décharge que pour les déchets ultimes à partir du $1^{\text {er }}$ juillet 2002 , impliquera donc un traitement préalable de ces solides pour un recyclage partiel. Afin de préparer cette évolution, une étude des aspects scientifiques et techniques du problème a été engagée dans le cadre du projet de recherche A5 du GARIH. En 1996, deux phases de cette étude pluriannuelle ont été réalisées :

- une première caractérisation de l'ensemble des sous-produits solides ;

- un recensement des filières de traitement opérationnelles ou pilotes.

The expression " sewerage solid by-products " designates all the solids produced by the operation and maintenance of streets and sewer systems, in the framework of an integrated approach of the urban wet weather discharges. As a part of the GARIH A5 research project, a specific study has been carried out in order to characterise the sewerage solid by-products (physico-chemical characteristics) and to assess their treatability (technologies, macro-economy...)

A literature review and a sampling study in Bordeaux have shown that the sewerage solid by-products are mainly mineral, with very low nutrient concentrations in comparison with sludge from waste water treatment plants, and with high pollutants loads, especially heavy metals and hydrocarbons. These solids, whose characteristics are highly variable from one site to another, are presently disposed of in landfills. According to the French law on waste products dated July 13th 1992, the future authorised landfills will only accept * ultimate waste products " after July 1st 2002. The sewerage solid by-products currently removed can not be considered as "ultimate waste products", and to comply with the regulation there will be a need to treat these solids in an attempt to recover as much material as possible for partial reuse.

The inventory of the present treatment processes and technologies, by literature review and visits of plants, led to the definition of a typical treatment process with four main steps : screening, hydrocycloning, classification and dewatering. This treatment gives washed sands that can be reused, and by-products that need to be further treated in existing devices. The technical tools are quite well identified, however their performances are up to now not precisely known. A detailed characterisation of all by-products generated by the treatment process is also necessary in order to better assess their treatability or reuse. 


\section{CARACTÉRISATION DES SOLIDES}

Une étude bibliographique préliminaire a montré que - peu d'études détaillées des sous-produits solides de l'assainissement ont été effectuées avec une définition claire des sites et des conditions de mesures, les produits ayant fait l'objet des mesures les plus nombreuses étant les produits de curage des collecteurs (voir tabl. I);

- les sous-produits solides de l'assainissement présentent des caractéristiques très variables d'un site à l'autre (voir tabl. 2).

Une campagne de prélèvements a donc été entreprise pour mieux connaître les caractéristiques de ces solides sur le ter- ritoire de la Communauté Urbaine de Bordeaux, où 21 sites de prélèvements ont été choisis (voir tabl. 3).

Les mesures effectuées sur les prélèvements sont regroupées dans le tableau 4.

Dans le cadre de ce court article, il n'est pas possible de commenter l'ensemble des résultats obtenus. Néanmoins, les principales conclusions sont que les sous-produits solides de l'assainissement sont essentiellement minéraux (voir figs. 1 et 2), pauvres en nutriments par rapport aux boues de stations d'épuration (les concentrations sont environ 10 fois plus faibles que dans les boues de stations qui contiennent en moyenne $30 \mathrm{~g} \mathrm{~N} / \mathrm{kg} \mathrm{MS}$ et $20 \mathrm{~g} \mathrm{P} / \mathrm{kg}$ MS : voir tabl. 2) et fortement chargés en métaux lourds (voir figs. 3 et 4 ) et en hydrocarbures (voir fig. 5).

Tableau 1. - Références bibliographiques validées pour l'étude des sous-produits solides.

\begin{tabular}{|c|c|c|}
\hline Provenance & $\begin{array}{l}\text { références } \\
\text { biblio. } \\
\text { retenues }\end{array}$ & Sources \\
\hline Voiries & 7 & $\begin{array}{c}\text { Artières (1987), IRH (1995b), Plan Urbain (1989), } \\
\text { Yamada et al. (1993), Hamilton et al. (1984), } \\
\text { Harrison (1979), Latimer et al. (1990) }\end{array}$ \\
\hline Avaloirs & 4 & Artières (1987), IRH (1995b), Desbordes (1994), IRH (1983) \\
\hline Collecteurs unitaires & 7 & $\begin{array}{c}\text { Artières (1987), IRH (1995b), Lassus et Salomé (1993), } \\
\text { IRH (1983), Desbordes (1994), Michelbach et al. (1992), } \\
\text { Flores-Rodriguez et al. (1993) }\end{array}$ \\
\hline Collecteurs eaux usées & 3 & IRH (1983), Flores-Rodriguez et al. (1993), Desbordes (1994) \\
\hline Collecteurs eaux pluviales & 3 & IRH (1983), IRH (1995b), Desbordes (1994) \\
\hline Bassins à sec & 4 & $\begin{array}{l}\text { Baladès et Bertrand-Krajewski (1995), Chebbo (1992), } \\
\text { Flores-Rodriguez et al. (1993), Paitry et Pautis (1985) }\end{array}$ \\
\hline Bassin en eau & 2 & Lappe (1986), Chopard (1989) \\
\hline
\end{tabular}

Tableau 2. - Synthèse des caractéristiques physico-chimiques des solides de différentes provenances.

\begin{tabular}{|c|c|c|c|c|c|c|c|c|}
\hline \multirow{2}{*}{\multicolumn{2}{|c|}{ Paramètres }} & \multirow{3}{*}{$\begin{array}{c}\text { Voiries } \\
\sim 90 \\
\end{array}$} & \multirow{3}{*}{$\begin{array}{c}\text { Avaloirs } \\
35-75 \\
\end{array}$} & \multicolumn{3}{|c|}{ Collecteurs } & \multicolumn{2}{|c|}{ Bassins } \\
\hline & & & & \multirow{2}{*}{$\begin{array}{c}\text { Unitaires } \\
65-80 \\
\end{array}$} & \multirow{2}{*}{$\begin{array}{c}\text { Eaux usées } \\
70 \\
\end{array}$} & \multirow{2}{*}{$\begin{array}{c}\begin{array}{c}\text { Eaux } \\
\text { pluviales }\end{array} \\
80-90\end{array}$} & \multirow{2}{*}{$\begin{array}{l}\text { A sec } \\
15-45 \\
\end{array}$} & \multirow{2}{*}{ En eau } \\
\hline MS & $\%$ & & & & & & & \\
\hline MO & $\%$ MS & $5-7$ & $5-25$ & $2-30$ & $0,5-85$ & $1,5-10$ & $15-25$ & 15 \\
\hline DCO & $\mathrm{mg} \mathrm{O} 2 / \mathrm{g} \mathrm{MS}$ & 70 & 140 & 165 & & & & \\
\hline СОТ & $\mathrm{g} / 100 \mathrm{~g}$ MS & 40 & & & & & 10 & \\
\hline NTK & $\mathrm{mg} \mathrm{N} / \mathrm{kg} \mathrm{MS}$ & 1 & $1-2,5$ & $1,5-3,5$ & 2,5 & 1 & & \\
\hline $\mathrm{P}$ total & $\mathrm{mg} P / \mathrm{kg} \mathrm{MS}$ & 0,7 & $0,5-1$ & $3,5-7,5$ & 15 & 3 & & \\
\hline $\mathrm{Cd}$ & $\mathrm{mg} / \mathrm{kg} \mathrm{MS}$ & $0,02-10,7$ & $1-8,8$ & $0,3-10,6$ & $0,1-7$ & $0,03-7,2$ & $2,2-795^{*}$ & \\
\hline $\mathrm{Cr}$ & $\mathrm{mg} / \mathrm{kg} \mathrm{MS}$ & $15-370$ & $20-75$ & $10-85$ & $7-160$ & 5-95 & 55 & 60 \\
\hline $\mathrm{Cu}$ & $\mathrm{mg} / \mathrm{kg} \mathrm{MS}$ & $10-237$ & $20-270$ & $25-830$ & 415 & $20-210$ & $50-550$ & $15-620$ \\
\hline $\mathrm{Hg}$ & $\mathrm{mg} / \mathrm{kg} \mathrm{MS}$ & $0,05-0,12$ & $0,05-1,9$ & $0,2-37,4$ & $0,4-201,4$ & $0-1,2$ & $10 * *$ & $0,1-0,4$ \\
\hline $\mathrm{Ni}$ & $\mathrm{mg} / \mathrm{kg} \mathrm{MS}$ & $5-60$ & $20-115$ & $15-220$ & 130 & 170 & 20 & 40 \\
\hline $\mathrm{Pb}$ & $\mathrm{mg} / \mathrm{kg} \mathrm{MS}$ & $50-6630$ & $165-1315$ & $15-1400$ & $18-960$ & $20-245$ & $300-885$ & $30-350$ \\
\hline $\mathrm{Se}$ & $\mathrm{mg} / \mathrm{kg} \mathrm{MS}$ & $<0,1$ & 0,35 & 0,15 & & & & \\
\hline $\mathrm{Zn}$ & $\mathrm{mg} / \mathrm{kg} \mathrm{MS}$ & $50-1600$ & $200-620$ & $150-10100$ & $380-770$ & $100-740$ & $380-3850$ & $50-840$ \\
\hline $\mathrm{HC}$ & $\mathrm{mg} / \mathrm{kg} \mathrm{MS}$ & 325 & $130-820$ & $400-700$ & & 70 & $1225-15700$ & \\
\hline
\end{tabular}

(*) forte concentration due à une pollution industrielle identifiée

$(* *)$ concentration anormalement élevée, probablement due à un rejet accidentel. 
Tableau 3. - Inventaire des 21 sites de prélèvements à Bordeaux.

\begin{tabular}{|c|c|c|}
\hline $\begin{array}{c}\text { Provenance } \\
\text { des prélèvements }\end{array}$ & Nombre d'échantillons & Type de zones \\
\hline Voirie & 2 au site de dépotage & Centre ville et Bordeaux rive droite \\
\hline Avaloir & 5 dont 1 au site de dépotage & $\begin{array}{c}2 \text { en centre ville, } 1 \text { en zone commerciale, } \\
\text { en zone industrielle et } 1 \text { hors de la ville }\end{array}$ \\
\hline Collecteur unitaire & 4 dont 2 au site de dépotage & Centre ville \\
\hline Collecteur séparatif & $\begin{array}{c}5 \text { dont } 2 \text { eaux usées } \\
\text { et } 3 \text { eaux pluviales }\end{array}$ & Rive gauche la ville \\
\hline Bassin de retenue & 5 dont 3 unitaires et 2 pluviaux & Rors de \\
\hline
\end{tabular}

Tableau 4. - Liste des paramètres étudiés et des méthodes de mesure.

\begin{tabular}{|c|c|c|}
\hline Paramètres & Méthode & Principe \\
\hline Granulométrie & NF P 18-560 & Tamisage et densimétrie \\
\hline Humidité/Matière sèche & NF T 90-105 & Séchage à $105{ }^{\circ} \mathrm{C}$ \\
\hline Matière organique/Minérale & NF T 90-029 & Calcination à $550{ }^{\circ} \mathrm{C}$ \\
\hline Carbone organique total & NF T 90-102 & Oxydation du C (par $\mathrm{K}_{2} \mathrm{Cr}_{2} \mathrm{O}_{7}$ ) en $\mathrm{CO}_{2}$ \\
\hline Hydrocarbures totaux & NF T 90-114 & \begin{tabular}{c} 
Spectrophotométrie infrarouge \\
\hline Cadmium, Cuivre, Plomb et Zinc
\end{tabular} \\
\hline
\end{tabular}

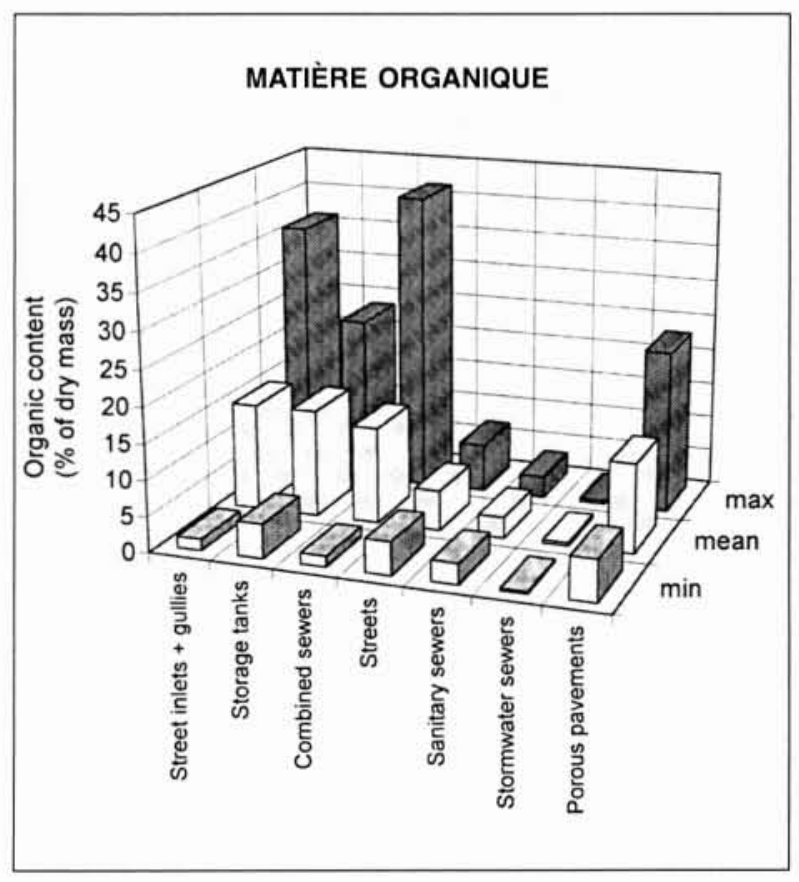

1. Teneur en matière organique en fonction de l'origine du prélèvement.

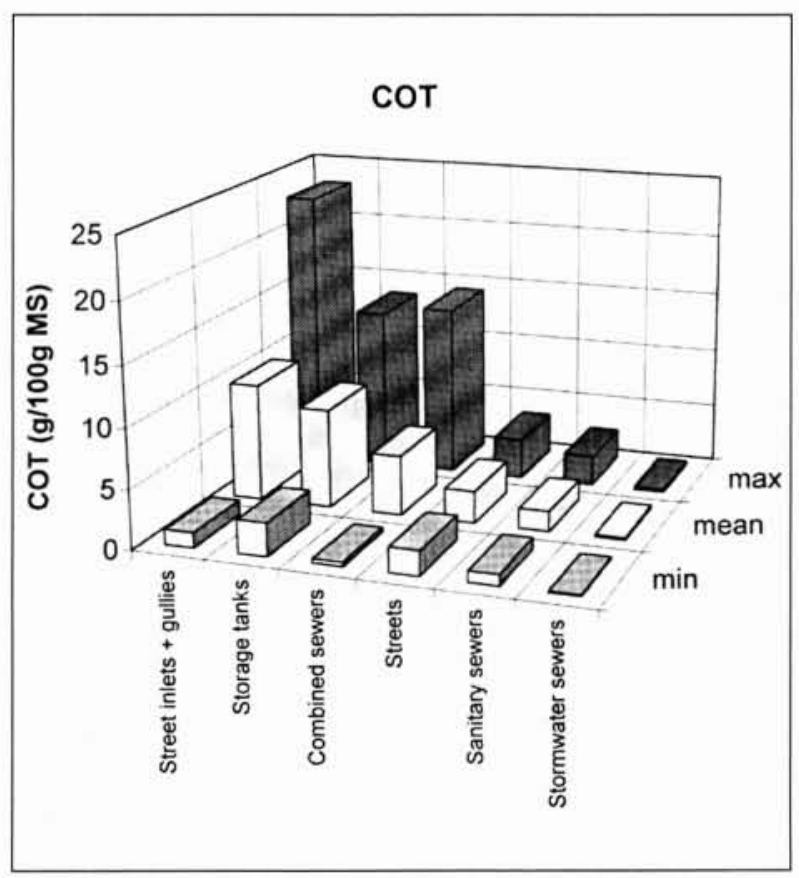

2. Concentration en COT en fonction de l'origine du prélèvement. 


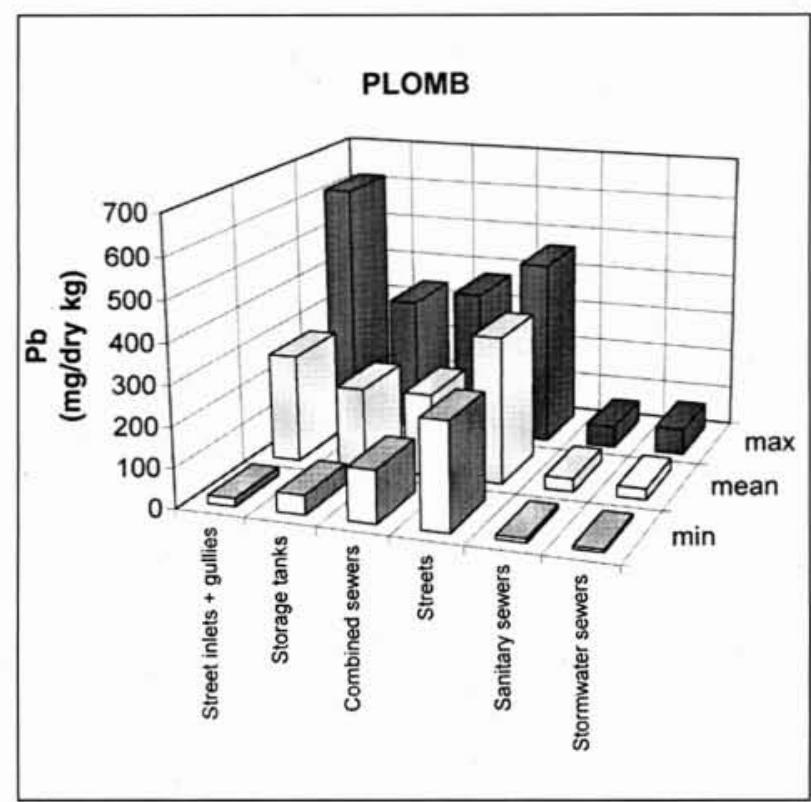

3. Concentration en plomb en fonction de l'origine du prélèvement.

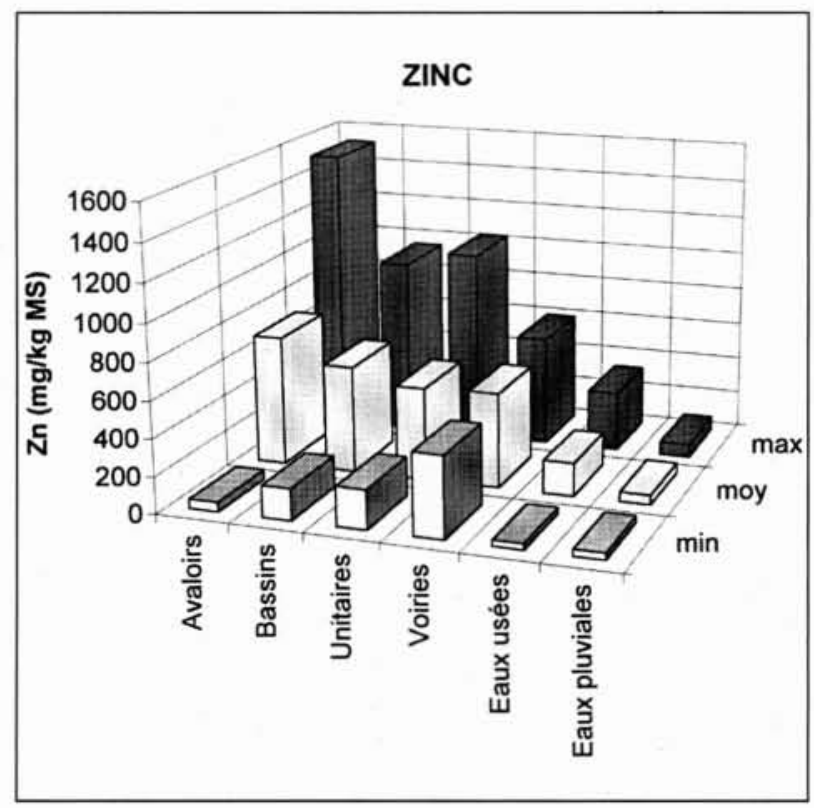

4. Concentration en zinc en fonction de l'origine du prélèvement.

Les courbes granulométriques sont également variables selon l'origine du sous-produit. Une indication de cette variabilité est donnée figure 6.

\section{III — FILIÈRE TYPE DE TRAITEMENT}

Le recensement des filières et des technologies de traitement (inventaire et visites sur sites) a permis de définir une filière

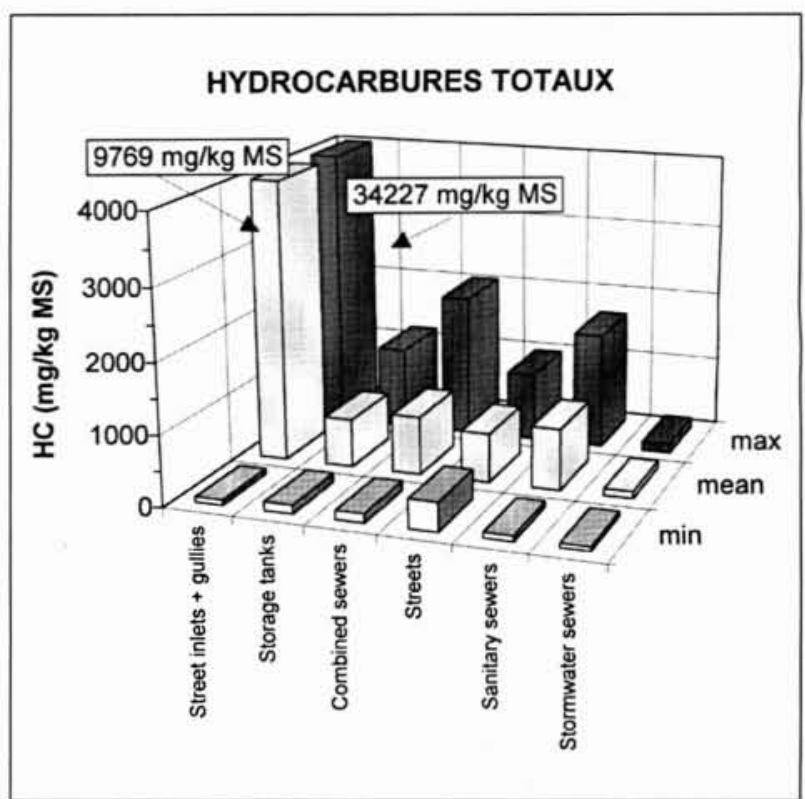

5. Concentration en hydrocarbures en fonction de l'origine du prélèvement.

type comprenant quatre étapes principales : criblage, lavage, classification et essorage (fig. 7). Ce traitement permet d'obtenir des sables lavés (environ 55-60\% de la masse de produits bruts introduits) utilisables en remblaiement de tranchées et des sous-produits (déchets grossiers et eaux de lavage) qui doivent rejoindre des chaînes de traitement existantes (incinération, compostage, traitement des eaux résiduaires, etc.). Toutefois, les performances exactes de ces techniques sont encore mal connues et une caractérisation fine des différents sous-produits obtenus au cours du traitement est nécessaire pour mieux évaluer leur filière de valorisation ou de traitement. Une étude expérimentale est donc envisagée à Bordeaux pour 1998-1999.

Notre étude a montré que, dans la plupart des cas, le critère retenu pour dimensionner et qualifier une installation de traitement portait sur la teneur en matière organique. En référence à la norme NF P 11-300 qui établit une «classification des matériaux utilisables dans la construction des remblais et des couches de forme d'infrastructure routière ", les fournisseurs proposent comme objectif une teneur en matière organique des sables lavés inférieure à $3 \%$, ce qui revient, implicitement, à comparer les sous-produits solides à des sols naturels. Or cette comparaison ne présente guère d'intérêt. Des teneurs en matière organique comprises entre 3 et 10-12\% sont tout à fait acceptables pour constituer des remblais sans risque d'instabilité mécanique. En revanche, les caractéristiques granulométriques des sables lavés ne sont jamais prises en compte, alors qu'elles peuvent jouer un rôle important dans la valorisation du matériau (problèmes liés à la traficabilité, à la stabilité mécanique, à la nécessité de recourir à des mélanges, etc.).

Les eaux de lavage contiennent des charges polluantes extrêmement élevées, qui nécessitent soit une attention particulière en cas de traitement en station d'épuration classique (impacts sur les filières eaux et boues, problèmes de toxicité et de valorisation agricole des boues, etc.), soit un prétraitement physico-chimique avant rejet dans le réseau d'assainissement (assimilation à un rejet industriel). 


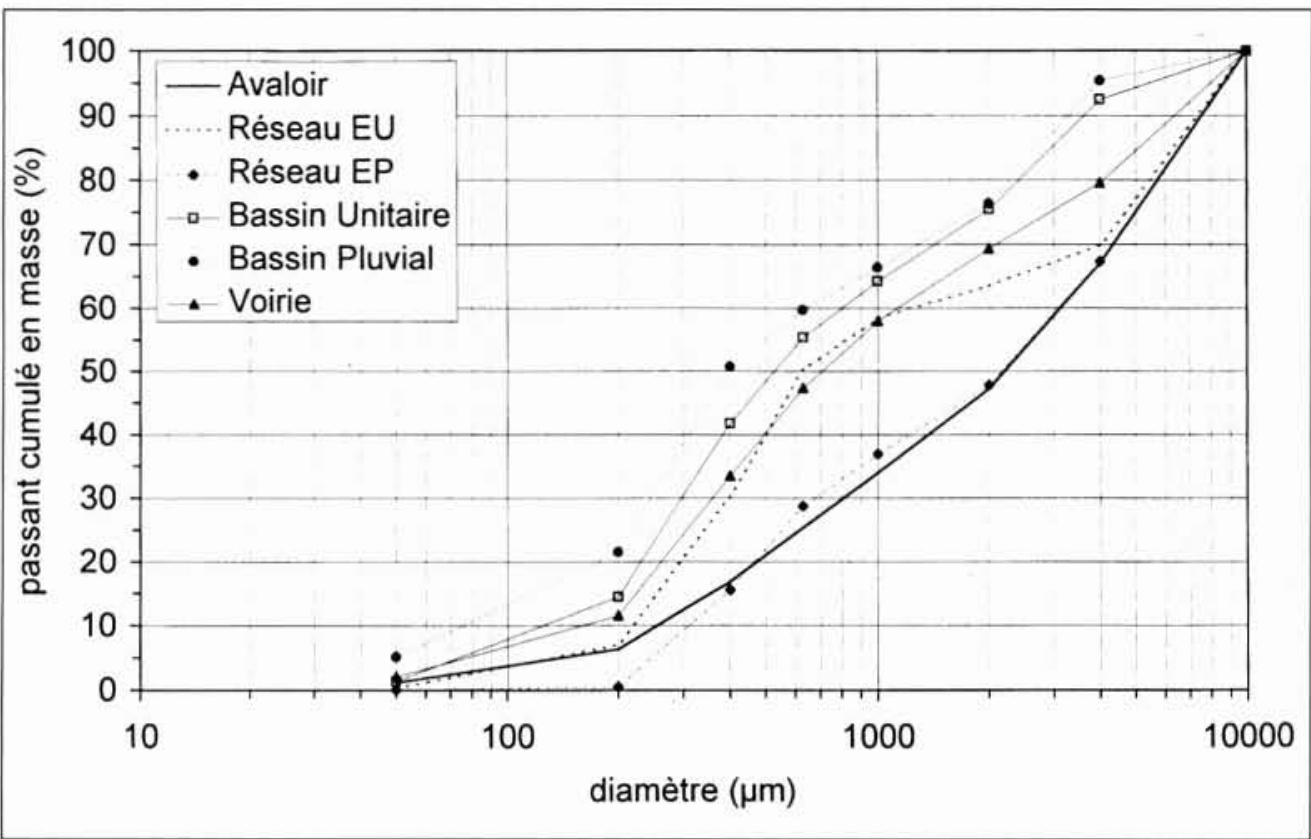

6. Courbes granulométriques moyennes par types de sous-produits.

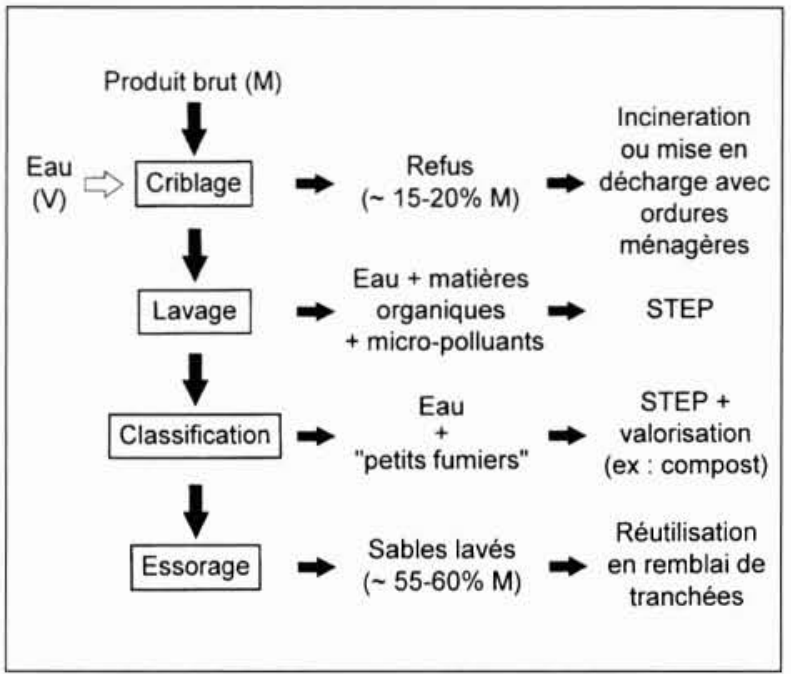

7. Filière type de traitement des sous-produits solides de l'assainissement.

\section{IV — PERSPECTIVES}

Les perspectives 1997-1999 pour cette étude sont les suivantes :

- des expérimentations sur pilote aux différentes étapes du traitement,

- une analyse technico-économique globale à l'échelle de la Communauté Urbaine de Bordeaux,

- une caractérisation complémentaire des solides par des tests de lixiviation (utilisation de la norme relative aux mâchefers qui paraît à ce jour la plus appropriée pour caractériser ces solides et leur valorisation en génie civil).

\section{BIBLIOGRAPHIE}

Delattre C., Bertrand-Krajewski J.-L. (1996) - Caractérisation et traitabilité des sous-produits solides de l'assainissement. Rapport intermédiaire 1996. Rapport de recherche GARIH/Lyonnaise des Eaux, Bordeaux, novembre 1996. 